\title{
Towards Environments That Have a Sense of Humor
}

\author{
Anton Nijholt \\ University of Twente, Human Media Interaction \\ P.O. Box 217, 7500 AE Enschede, The Netherlands \\ a.nijholtautwente.nl
}

\begin{abstract}
Humans have humorous conversations and interactions. Nowadays our real life existence is integrated with our life in social media, videogames, mixed reality and physical environments that sense our activities and that can adapt appearance and properties due to our activities. There are other inhabitants in these environments, not only human, but also virtual agents and social robots with which we interact and who decide about their participation in activities. In this paper we look at designing humor and humor opportunities in such environments, providing them with a sense of humor, and able to recognize opportunities to generate humorous interactions or events on the fly. Opportunities, made possible by introducing incongruities, can be exploited by the environment itself, or they can be communicated to its inhabitants.
\end{abstract}

Keywords: humor, intelligent environments, computational humor, sensors, actuators, virtual reality, videogames, virtual agents, social robots.

\section{Introduction and Motivation}

Humor is important in our daily activities. Although we sometimes may tell jokes and listen to them, most of the humor we experience is in conversations, in behavioral activity and in actions and situations in general. And, unlike the joke telling and listening experience, in humor that appears spontaneously, we play an active role in letting this humor come into existence. We assess a situation and decide whether it contains the elements to construct a humorous remark and whether it is appropriate to generate such a remark. We assess a situation and we decide whether our next activity allows a humorous effect or whether it should allow others to introduce a humorous act. This spontaneous humor depends on context, history of activities and interactions.

Today, sensor-equipped environments can be designed to sense and interpret human activity and interaction. Intelligent environments can interpret and support human-human interaction, but they can also be inhabited by social (virtual) agents and by social robots. These environments and their artificial inhabitants can play an active role in generating (potential) humorous situations and interactions. Actuators can make (unexpected and incongruous) changes to the environment. The environment can distribute knowledge about what can be done to its human or artificial agents who can then play a role in generating incongruous situations and interactions.

D. Reidsma et al. (Eds.): INTETAIN 2014, LNICST 136, pp. 77-80, 2014.

(C) Institute for Computer Sciences, Social Informatics and Telecommunications Engineering 2014 
In contrast, when we look at humorous texts, a movie or a comedy, the humorous situations and interactions are constructed in advance. Readers or audience members don't play an active role, and although there are exceptions, we are expected to consume rather than to be creative and construct or co-construct humor. Between the sensor-equipped physical environments and media such as texts, stage performances and movies we can position virtual and augmented reality worlds that simulate aspects of the real world in order to meet entertaining or educational challenges presented to us. These virtual and augmented worlds allow interaction, but the interaction is guided and controlled by the application.

This is not different from what we encounter in videogames, although there we only see mouse, keyboard and joystick interactions. This changed with the advent of devices such as the Kinect and the Wii remote controller. In videogames we have fullfledged simulations of real or fantasy worlds, inhabited with virtual agents (avatars), where these agents represent (semi-) autonomous users or game generated characters. Humor is present in videogames. Humor is available in dialogues between game characters, comments on a gamer's behavior and actions, incongruous characters, jokes and incongruous introductions in cut scenes, situations that challenge the gamer by employing non-Euclidean geometry or unusual physics or humor is available in a meta-channel of a multi-user role playing game that allows gamers to employ textual or speech chat in order to discuss strategies, to negotiate or to comment.

The convergence of real and constructed worlds (virtual worlds, videogames) made possible by sensors and actuators, new display facilities, wearables and humanoid partners makes it highly appropriate to identify a research area that is concerned with humor design or the design of potentially humorous situations that can be exploited, when desired and appropriate, by the inhabitants of these environments.

\section{Humor, Humor Theory, Humor Computation: State of the Art}

Can we have computer intelligence that can both recognize humor and adequately respond to it? Or that can create humor at the right time? To do so we need humor models that are sufficiently formal to extract rules for understanding and generating humor, rules which a computer algorithm can process and which also allow taking into account the context of interpretation and generation. That is not all it takes either. To understand humour, it requires the knowledge of a certain amount of 'common sense'. What is normal, and what is different? It requires knowledge of global affairs. Perhaps we should just accept that modeling such knowledge, and reasoning about it, is a hopeless task. People are able to make - and understand - the most ridiculous associations. On the other hand, we have also seen that a computer, with the help of sheer computing power and relatively simple modeling, can beat a world chess champion or win a game like ‘jeopardy' against human opponents. However, in both of those instances, this follows decades of research into playing strategies, reasoning, learning, retrieval, forming associations, processing natural language and modeling to represent knowledge. Sadly, for whatever reason, we have not seen any similar work on detecting or generating humor and potential humorous situations. 
There exist theories of humor. They can be found in many textbooks or articles on humor, e.g., [1,2]. They overlap and focus on different aspects (social, emotion, cognition) of humor. In superiority or contempt theory we laugh about the misfortune of others. Social and sexual taboos play a role in Freud's observations on humor. We can speak of 'cognitive taboos' in jokes that defy logic. Bergson [3] drew attention to 'inelasticity' of movement and behavior that creates comical situations. Kant, Schopenhauer and Koestler were among the many humor observers that focused on the role of incongruity and its resolution in humor. Although often incongruity is seen as a necessary condition for humor, it certainly is not a 'sufficient' condition. When is an incongruity funny? In addition to attempts to find such conditions, conditions have been defined that address the state of mind in which one is receptive to humor [4].

There are exceptions, but the mainstream of humor research that seems promising from a point of view of leading to formal models, is on verbal humor. Formalizing verbal humor is part of computational linguistics and its extensions in artificial intelligence (common sense and world knowledge representation and reasoning, machine learning). This is a relatively successful area of research, hence we see much interest in detecting and analyzing humor in verbal jokes, texts, and conversations $[1,5,6,7]$. Although some of the theories of humor contain elements that go beyond linguistics, with the exception of Bergson, humor in the physical world is hardly addressed, and certainly not from the point of view of a possible formalization that can be used to embed humor creation and facilitation in intelligent environments.

\section{Humor in Natural and Artificial Worlds}

Incongruities appear or can be introduced in the real world. Many humor techniques in the context of the real world were identified by Morreal [8]. When do we laugh about things that are happening in the real world? Obviously, we cannot discount the role of language that accompanies our real-world activities and interactions. Nevertheless, in [8] we find an attempt to systematically list of kinds of humor in the real world. His categories range from physical imperfections of people to thoughtlessness and stupidity, moral flaws, failed actions, imitating, repetition, and coincidence. Many of these categories or elements of these categories can be found also in observations on humor in advertisements, in comedy, and in movies. Greek plays have been analyzed for their use of humor. Obviously, humor techniques in comedy have been well described [9]. Less known are analyses in movies [10], and audiovisual data (advertisements) [11]. In [10] we see many examples of sight gags, that is, situations where movie directors play with interpretations. This can be done from the perspective of the viewers of the film or from the characters in the film, and the viewer being or becoming aware of it. Obviously, in animated movies we see lots of incongruities, that is, breaking laws of physics, biology, and social norms. In [11] it is shown how a glossary of verbal humor techniques [12] can be used in the audiovisual domain. Using different modalities and in particular cross-modal incongruities provides another view on humor creation in real or simulated worlds. Humorous products [13] then become part of environments with a sense of humor. Cross-modal ambiguities or incongruities can as well introduced in interactions with real and artificial agents that inhabit environments with a sense of humor. 
We can try to introduce such incongruities in the domain of intelligent environments in general, including videogames. In fact, they can already been found in many videogames, often in pre-canned ways, without knowledge or active participation of the gamer. But there are attempts to model humor in the context of videogames too [14]. This is more difficult than in texts, in advertisements or on stage using pre-canned humor, without participation of the audience. It will be more difficult when indeed interaction with human participants is pursued. With advanced AI and agent technologies possibilities arrive. With present sensor and actuator technology it is certainly worthwhile to investigate possibilities to steer a physical environment and his inhabitants to (potentially) funny situations.

\section{Conclusions}

In this short paper we have looked at the possibility of modeling humor: humor in language and speech, humor in everyday life and humor in audio visual worlds such as film, commercials and video games. The essence of the arguments put forward was that sensors and actuators are making it possible that the future worlds we will live in know about humor. They can detect humorous events and can anticipate and facilitate humorous events. This article is meant to identify the challenges that have to be met.

\section{References}

1. Raskin, V. (ed.): The Primer of Humor Research. Mouton de Gruyter, Berlin (2008)

2. Mulder, M.P., Nijholt, A.: Humour Research: State of the Art. Technical report. CTIT, University of Twente, the Netherlands (2002)

3. Bergson, H.: Laughter. An essay on the meaning of the comic. Translated from Le Rire. Essai sur la signification du comique 1900, Gutenberg project (2003)

4. Apter, M.J.: The experience of motivation: The theory of psychological reversals. Academic Press, London (1982)

5. Nijholt, A.: Conversational Agents and the Construction of Humorous Acts. In: Nishida, T. (ed.) Conversational Informatics: An Engineering Approach, pp. 21-47. John Wiley \& Sons, Chicester (2007)

6. Tinholt, H.W., Nijholt, A.: Computational Humour: Utilizing Cross-Reference Ambiguity for Conversational Jokes. In: Masulli, F., Mitra, S., Pasi, G. (eds.) WILF 2007. LNCS (LNAI), vol. 4578, pp. 477-483. Springer, Heidelberg (2007)

7. Mihalcea, R.: The Multi-disciplinary Facets of Research on Humour. In: Masulli, F., Mitra, S., Pasi, G. (eds.) WILF 2007. LNCS (LNAI), vol. 4578, pp. 412-421. Springer, Heidelberg (2007)

8. Morreal, J.: Taking Laughter Seriously. State University of New York Press, Albany (1983)

9. Byrne, J.: Writing Comedy. A \& C Black Publishers, London (1999)

10. Carroll, N.: Theorizing the Moving Image. Cambridge University Press, Cambridge (1996)

11. Buijzen, M., Valkenburg, P.M.: Developing a Typology of Humor in Audiovisual Media. Media Psychology 6(2), 147-167 (2004)

12. Berger, A.A.: An Anatomy of Humor. Transaction Publishers, New Brunswick (1993)

13. Ludden, G.D.S., Kudrowitz, B.M., Schifferstein, H.N.J., Hekkert, P.: Surprise and humor in product design. Humor 25(3), 285-309 (2012)

14. Dormann, C., Boutet, M.: Incongruous avatars and hilarious sidekicks: Design patterns for comical game characters. Paper \#58, Proceedings DiGRA 2013, Atlanta, GA, USA (2013) 